\title{
Leiomyoma with Granulomatous Inflammation: Tissue Confirmation of Sarcoidosis
}

\author{
Kimi Verilhac' ${ }^{1}$ Jaime Arruda ${ }^{2}$, Miriam D. Post ${ }^{1}$ \\ ${ }^{1}$ Department of Pathology, University of Colorado Anschutz Medical Campus, Aurora, USA \\ ${ }^{2}$ Department of Obstetrics and Gynecology, University of Colorado Anschutz Medical Campus, Aurora, USA \\ Email: Miriam.Post@ucdenver.edu
}

Received 20 September 2013; revised 20 October 2013; accepted 28 October 2013

Copyright (C) 2014 by authors and Scientific Research Publishing Inc.

This work is licensed under the Creative Commons Attribution International License (CC BY). http://creativecommons.org/licenses/by/4.0/

(c) (i) Open Access

\begin{abstract}
Granulomatous inflammation of the uterus is rare, and sarcoidosis involving the uterus is even less common. Sarcoidosis is an idiopathic granulomatous disease that can affect any organ, but most typically involves the lungs, lymph nodes, spleen, liver, skin, and eye. Although it can be diagnosed clinically based on a constellation of symptoms, definitive diagnosis typically requires demonstration of non-infectious non-caseating granulomas on tissue biopsy. It is a diagnosis of exclusion, and other causes of granulomatous inflammation must be excluded. We report here a case of granulomatous inflammation involving a leiomyoma in a patient with a previous clinical diagnosis of sarcoidosis. This was the patient's first tissue-based diagnosis of sarcoidosis and highlighted the importance of adequate sampling of routine surgical specimens, as they may harbor signs of systemic disease.
\end{abstract}

\section{Keywords}

Sarcoidosis; Leiomyoma; Granulomatous Inflammation

\section{Introduction}

Sarcoidosis is a relatively rare disease, affecting approximately 10 - 71 per 100,000 people in the United States. It is usually sporadic and idiopathic, although familial clustering has been reported. Women are affected more often than men and most commonly present between 20 and 40 years of age, often with a cough, fever, weight loss and chest pain. The diagnosis can be made presumptively based on clinical features, however definitive diagnosis requires a tissue specimen demonstrating non-caseating granulomas, where other etiologies for the 
granulomatous inflammation have been excluded.

\section{Report of a Case}

A 36 years old G1 P0010 African American woman presented to her gynecologist with pelvic pressure, constipation, and obstructive defecation. Her medical history was significant for sarcoidosis, obesity, and remote syphilis and Chlamydia infections. On physical examination, she had a $16-18$ weeks size uterus which was fixed in the pelvis. On ultrasound, her uterus measured $16.7 \mathrm{~cm}$ in length $\times 12.5 \mathrm{~cm}$ in width $\times 4.5 \mathrm{~cm}$ AP, with multiple well-circumscribed hypoechoic masses in the myometrium. The patient had previously been diagnosed with Lofgren syndrome in 2009, based on the clinical presentation of fevers, joint pain, erythema nodosum, and hilar lymphadenopathy (Figure 1(A)). She was treated with steroids for one year, and had since been off therapy with no signs of disease activity. During her gynecologic preoperative visit, she had a normal pulmonary exam and normal oxygen saturation, and a recent CT scan showed near complete interval resolution of her mediastinal and hilar adenopathy.

The patient subsequently underwent a robotic-assisted laparoscopic myomectomy for symptomatic relief. This was converted to an open laparotomy after finding a large parasitic fibroid posterior to the uterus with a shared blood supply from the uterus and the sigmoid colon mesentery. Her postoperative course was complicated by intraperitoneal bleeding from the bowel mesentery and a reoperation for repair of the bleeding site. She also received a blood transfusion. During her recovery from surgery, she had a persistent oxygen requirement, attributed to sarcoidosis and possible obstructive sleep apnea. She had a perfusion scan to rule out a pulmonary embolism which was negative.

$732 \mathrm{~g}$ of fragmented uterine fibroids were received in pathology, measuring $15.5 \times 15 \times 4 \mathrm{~cm}$ in aggregate. Gross examination revealed whorled pink-white cut surfaces, without areas of necrosis or hemorrhage. The specimen was thoroughly sampled, with approximately one section per centimeter submitted for processing. Histologic examination revealed fragments of benign leiomyoma, with a typical pattern of interlacing fascicles of smooth muscle bundles (Figure 1(B)). Two sections demonstrated areas of granulomatous inflammation within the leiomyoma, characterized by tight clusters of epithelioid cells admixed with abundant multinucleated giant cells (Figures 1(C) and (D)). There were no areas of necrosis, and AFB and GMS stains were negative for organisms. The case was signed out as fragments of benign leiomyoma with focal granulomatous inflammation, consistent with the patient's clinical history of sarcoidosis. This was the patient's first tissue diagnosis of sarcoidosis.

\section{Discussion}

Uterine leiomyomas are benign smooth muscle tumors, more commonly known as fibroids, and comprise a substantial proportion of surgical specimens from gynecologic surgeries. They are the most common uterine neoplasm, although the true incidence is uncertain as many are asymptomatic and remain undiagnosed. By the age of 50 , leiomyomas are found in the uterus of almost $70 \%$ of white women and more than $80 \%$ of African American women [1]. The relative risk for leiomyomas is two to three times greater in Black than Caucasian women, and the average age at diagnosis in black women is four years younger [2]. Genetic risk factors also contribute to familial patterns of inheritance. Although many leiomyomas are asymptomatic, some can produce significant symptoms including abnormal uterine bleeding, infertility or recurrent pregnancy loss, pain, pelvic pressure, and less commonly urinary incontinence or constipation. Symptomatic leiomyomas account for 30\% $40 \%$ of the approximately 600,000 hysterectomies performed annually in the United States. Treatment options are varied and include everything from expectant management to medical management to surgical intervention. Medical options include gonadotropin-releasing hormone agonists (Depo Lupron), oral contraceptive pills or progestins to control bleeding symptoms, and aromatase inhibitors. Procedural interventions include myomectomy, hysterectomy, uterine artery embolization or occlusion, and methods such as ablation or myolysis (with freezing, heating, laser, radiofrequency, or ultrasound techniques) [1].

Sarcoidosis is an idiopathic granulomatous disease that can affect any organ, with a prognosis which can range from benign to life-threatening. The age-adjusted incidence in the United States is $10-35$ per 100,000, with up to 71 per 100,000 in African American women. Familial clustering is reported in $5 \%-19 \%$ of patients, with polygenic, complex inheritance patterns. Sarcoidosis typically presents in patients between the ages of 20 40 years and is slightly more common in women than men [3]. Although sarcoidosis can involve any organ, it 

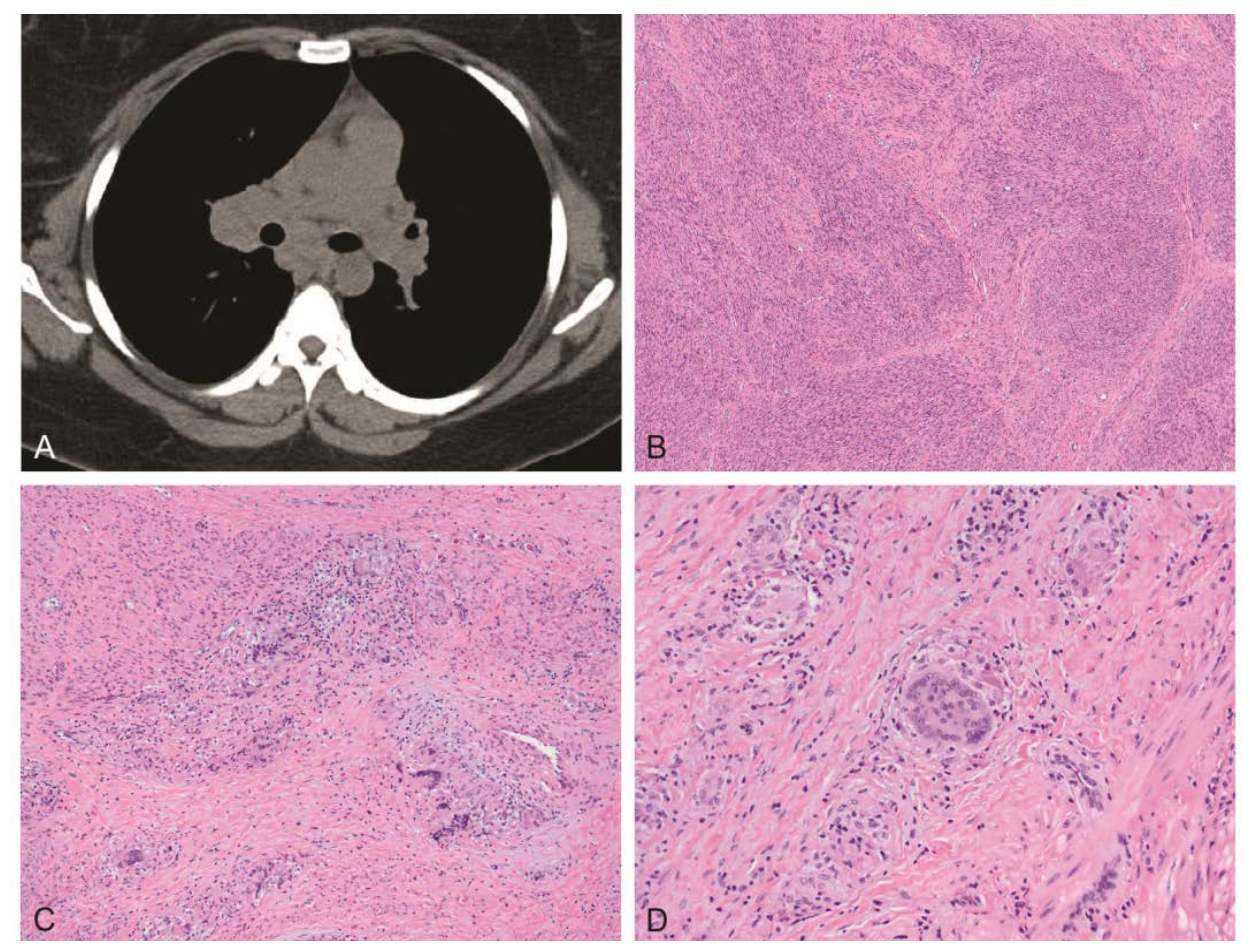

Figure 1. A. Chest CT scan showing bilateral hilar and mediastinal lymphadenopathy, consistent with sarcoidosis (routine ILD protocol without contrast). B. Benign leiomyoma with intersecting fascicles of bland smooth muscle bundles. C. Granulomatous inflammation within the leiomyoma. D. Higher power view demonstrating noncaseating granulomas with tight clusters of epithelioid cells admixed with multinucleated giant cells (Hematoxylin-eosin, original magnifications $40 \times[B], 100 \times[C], 200 \times[D])$.

typically affects the lungs, lymph nodes, spleen, liver, skin, and eye. Common signs and symptoms of sarcoidosis include cough, fever, weight loss, chest pain, painful ankle swelling, erythema nodosum, lupus pernio (a distinct mucocutaneous rash), and eye pain or blurred vision. There may be palpable lymphadenopathy, parotid enlargement, hepatosplenomegaly, or neurologic findings. Lung examination is usually normal. A common presentation of acute sarcoidosis is Lofgren syndrome, which consists of fever, hilar lymphadenopathy, ankle swelling, and erythema nodosum. If all these features are present, there is a 95\% diagnostic specificity for sarcoidosis [3]. Cardiac sarcoidosis is diagnosed in only 5\% of patients, but is important due to the significant morbidity and mortality.

Sarcoidosis is a diagnosis of exclusion. Many diseases present with findings similar to those of sarcoidosis, thus the diagnosis typically requires demonstration of non-caseating granulomas on biopsy. Other causes of granulomatous inflammation must, of course, be excluded. The risks and benefits of biopsy should be considered and those patients with classical Lofgren syndrome or asymptomatic bihilar lymphadenopathy may be presumptively diagnosed based on clinical features. Other tests can be used, but are less specific. These include bronchoalveolar lavage (finding of lymphocytosis with elevated CD4 - CD8 ratio is supportive of sarcoidosis), serum testing for angiotensin-converting enzyme levels (elevated in sarcoidosis), and gallium scanning, which will show the "panda sign" (lacrimal and parotid uptake) or the "lambda sign" (right paratracheal and hilar uptake). Chest X-ray is the recommended routine imaging, as $90 \%$ of patients have intrathoracic disease, most often bihilar or mediastinal lymphadenopathy (75\%) or pulmonary infiltrates (50\%) [3].

Histologically, tissues involved by sarcoidosis show well-formed non-caseating granulomas. These granulomas are composed of tightly clustered epithelioid cells, often with Langhans or foreign body-type giant cells. Central necrosis is unusual, however Schaumann bodies (laminated concretions of calcium and protein) or asteroid bodies (stellate inclusions) enclosed within giant cells are present in approximately $60 \%$ of cases. Over time, granulomas may become encased within fibrous rims or replaced by hyaline scars. Erythema nodosum will show panniculitis without granulomas. 
Although rare, sarcoidosis has been previously reported in the uterus. The clinical presentation is nonspe-cific, including recurrent pelvic pain, uterine bleeding, infertility, and persistent vaginal discharge [4]. Most cases report clinical presentations of abnormal uterine bleeding, with histologic findings of sarcoid granulomas in the endometrium or myometrium on either endometrial biopsy or hysterectomy [5]-[12]. One case reports isolated sarcoidosis in an endometrial polyp which was found incidentally on ultrasound [13]. There has been only one previously reported case of sarcoidosis within a leiomyoma [14] and none associated with such severe symptomatology as in the presented patient.

Granulomatous inflammation of the uterus in general is rare. Two studies reviewed cases of uterine granulomas; one found 30 cases over a ten year period [5], while the other found only 11 cases over a 20 year period [4]. The differential diagnosis for granulomatous uterine inflammation in the uterus includes infection (mycobacterial, fungal, viral, parasitic), foreign-body giant cell reaction (intrauterine devices, parasites, foreign material such as Monsel's solution), previous instrumentation or surgery, sarcoidosis, vasculitis, ceroid accumulation (granular pigment within macrophages, often associated with previous hemorrhage or necrosis), radiotherapy, and idiopathic granulomas [4]. Some leiomyosarcomas contain large numbers of giant cells [2], however these contain overtly malignant smooth muscle. Both studies found that the majority of their cases were due to previous instrumentation, and that the association with infection or systemic granulomatous diseases was rare in the uterus [4] [5].

This patient presented for surgical management of symptomatic fibroids and had an incidental finding of granulomatous inflammation. Infectious causes of inflammation were effectively ruled out by negative AFB and GMS stains, although a viral cause was not explicitly excluded. The patient did have an intrauterine device, but a foreign body reaction is unlikely due to the subserosal and intramural location of the granulomas. There was no evidence of vasculitis or ceroid accumulation, and the patient did not have a history of radiotherapy. Given the patient's clinical history of Lofgren's syndrome, sarcoidosis involving a leiomyoma is the most likely diagnosis. This was the patient's first histologic diagnosis of her clinical disease.

Sarcoidosis within the female genital tract is a relatively rare finding, and even rarer within leiomyomas. Only one case of sarcoidosis within a leiomyoma has been previously documented in the literature, though given the relative frequency of fibroids and sarcoidosis, one could presume that more cases exist and are undetected due to limited sampling of myomectomy specimens. This case illustrates the importance of adequately sampling leiomyomas, as these specimens may harbor signs of systemic disease.

\section{References}

[1] Munro, M.G. (2011) Uterine Leiomyomas, Current Concepts: Pathogenesis, Impact on Reproductive Health, and Medical, Procedural, and Surgical Management. Obstetrics \& Gynecology Clinics of North America, 38, 703-731. http://dx.doi.org/10.1016/j.ogc.2011.09.006

[2] Robboy, S., Mutter, G.L., Prat, J., Bentley, R.C., Russell, P. and Anderson, M.C. (2008) Uterine Smooth Muscle Tumors. Robboy's Pathology of the Female Reproductive Tract. 2nd Edition, Churchill Livingstone Elsevier, 457-484.

[3] O’Regan, A. and Berman, J.S. (2012) Sarcoidosis. Annals of Internal Medicine, 156, ITC5-1-15.

[4] Hoff, E. and Prayson, R.A. (2002) Incidental Granulomatous Inflammation of the Uterus. Southern Medical Journal, 95, 884-888. http://dx.doi.org/10.1097/00007611-200208000-00019

[5] Almoujahed, M.O., Briski, L.E., Prysak, M., Johnson, L.B. and Khatib, R. (2002) Uterine Granulomas: Clinical and Pathologic Features. American Journal of Clinical Pathology, 117, 771-775. http://dx.doi.org/10.1309/QFKL-WRWB-KYFE-KX2E

[6] Neumann, G., Rasmussen, K.L. and Olesen, H. (2002) Premenopausal Metrorrhagia as a Symptom of Sarcoidosis. European Journal of Obstetrics \& Gynecology and Reproductive Biology, 104, 171-173. http://dx.doi.org/10.1016/S0301-2115(02)00112-4

[7] Sherman, M.D., Pince, K.J. and Farahmand, S.M. (1997) Sarcoidosis Manifesting as Uveitis and Menometrorrhagia. American Journal of Ophthalmology, 123, 703-705.

[8] Pearce, K.F. and Nolan, T.E. (1996) Endometrial Sarcoidosis as a Cause of Postmenopausal Bleeding. A Case Report. The Journal of Reproductive Medicine, 41, 878-880.

[9] Sandvei, R. and Bang, G. (1991) Sarcoidosis of the Uterus. Acta Obstetricia et Gynecologica Scandinavica, 70, 165167. http://dx.doi.org/10.3109/00016349109006202

[10] DiCarlo, F.J., Jr., DiCarlo, J.P., Robboy, S.J. and Lyons, M.M. (1989) Sarcoidosis of the Uterus. Archives of Pathology \& Laboratory Medicine, 113, 941-943. 
[11] Honore, L.H. (1981) Asymptomatic Genital Sarcoidosis. Australian and New Zealand Journal of Obstetrics and Gynaecology, 21, 188-190. http://dx.doi.org/10.1111/j.1479-828X.1981.tb00918.x

[12] Ho, K.L. (1979) Sarcoidosis of the Uterus. Human Pathology, 10, 219-222. http://dx.doi.org/10.1016/S0046-8177(79)80009-X

[13] Rosa e Silva, J.C., de Sa Rosa e Silva, A.C., Aguiar, F.M., Poli Neto, O.B., Candido dos Reis, F.J. and Nogueira, A.A. (2006) Isolated Endometrial Polypoid Sarcoidosis in a Post-Menopausal Patient: Case Report. Maturitas, 53, 489-491. http://dx.doi.org/10.1016/j.maturitas.2005.12.007

[14] Menzin, A.W., You, T.T., Deger, R.B., Brooks, J.S. and King, S.A. (1995) Sarcoidosis in a Uterine Leiomyoma. International Journal of Gynecology \& Obstetrics, 48, 79-84. http://dx.doi.org/10.1016/0020-7292(94)02240-2 